\title{
Editorial for annual special issue on posterior fossa tumors
}

\author{
Paul Steinbok $^{1} \cdot$ Michel Zerah ${ }^{2,3}$
}

Received: 23 July 2015 / Accepted: 26 July 2015

(C) Springer-Verlag Berlin Heidelberg 2015

The focus of this annual special issue is posterior fossa tumors in children. There are a number of reasons to choose a unique theme for publication in a special issue. The most obvious is when a new field develops (e.g., antenatal neurosurgery) or when new progress or understanding has occurred in a narrow field of interest (e.g., cortical dysplasia in children or treatment of craniopharyngiomas). However, it is also important from time to time to have an up-to-date appraisal on a common topic (e.g., hydrocephalus, craniosynostosis) in which we all have extensive knowledge but in which new trends, sometimes at the limit of our field of competence, make such an update valuable.

Posterior fossa tumors are clearly in this latter category. They are part of our daily practice and thousands of them are treated all around the word in any pediatric neurosurgical unit. However, advanced neuroimaging, developments in molecular genetics, the use of intraoperative neurophysiology, and better understanding of longer-term results have changed our management of these common tumors.

This special issue covers the different types of posterior fossa tumors, common (medulloblastoma, ependymoma, cerebellar astrocytoma, brain stem tumor) or less common and less discussed (CriNET, PNET, CP angle tumors), and focuses on specific and sometimes controversial surgical aspects of these tumors (biopsy

Paul Steinbok

psteinbok@cw.bc.ca

1 Division of Neurosurgery, Department of Surgery, University of British Columbia and BC Children's Hospital, Vancouver, BC, Canada

2 Department of Pediatric Neurosurgery, Necker Enfants Malades Hospital. Assistance Publique Hopitaux de Paris. APHP, 149 rue de Sèvres, 75015 Paris, France

3 Université Paris Descartes, Sorbonne, Paris Cité, France in DIPG, surgical approaches of the brain stem, intraoperative monitoring, treatment of hydrocephalus). The value of neuroimaging of these tumors is also reviewed.

Most importantly, we wished to address in detail a number of topics for which our understanding has improved dramatically in the last 10 years and pushed us to completely reconsider the treatment of posterior fossa tumors in children. Firstly, the new biological understanding of the nature of these tumors has led to new classifications, new prognostic factors, and new protocols of treatment, some of which have already been implemented and others that are in development for future management. Secondly, with the increased number of long-term survivors of patients with posterior fossa tumors, it is obvious that the quality of life is a crucial aspect of the management of these tumors. Thirdly, our understanding of the importance of the cerebellum not only in motor function but also in higher cortical function has become better recognized and in this issue, cerebellar mutism and all the long-term neuropsychological problems after posterior fossa tumor surgery and their possible solutions are extensively discussed.

We wish to thank all the authors for their tremendous contributions, and we hope that you will have the same interest and pleasure to read this special issue as we have had to edit it. 